\title{
Recurrent Vulvar Carcinoma
}

National Cancer Institute

\section{Source}

National Cancer Institute. Recurrent Vulvar Carcinoma. NCI Thesaurus. Code C9054.

The reemergence of vulvar carcinoma after a period of remission. 